\title{
Dacriocistografia na propedêutica da via lacrimal excretora de adultos
}

\author{
Dacryocystography in adult lacrimal system evaluation
}

\author{
Silvana Artioli Schellini ${ }^{1}$ \\ Laura A. Hercules ${ }^{2}$ \\ Carlos RobertoPadovani ${ }^{3}$ \\ Sammy Mendes do Nascimento ${ }^{4}$ \\ Paula Sian Lopes ${ }^{4}$ \\ Ricardo de Campos Schellini ${ }^{5}$
}

\begin{tabular}{|l|}
\hline RESUMO \\
\hline Objetivo: Avaliar os achados dacriocistográficos em indivíduos suspeitos \\
de obstrução de vias lacrimais excretoras. Métodos: Oestudo foi retrospecti- \\
vo, tendo sido avaliadas as dacriocistografias de 100 indivíduos adultos, \\
suspeitos de obstrução nasolacrimal, atendidos na Faculdade de Medicina \\
de Botucatu - UNESP. Os dados obtidos foram submetidos à análise \\
estatística descritiva e teste do qui-quadrado. Resultados: A maioria dos \\
pacientes era do sexo feminino e maiores de 60 anos, apresentando obstru- \\
ção baixa, localizada no seio de Arlt, com saco lacrimal graus 2 ou 3. Vias \\
lacrimais pérvias, com e sem dilatação, também foram encontradas, assim \\
como alterações nasais, como hipertrofia de cornetos. Conclusão: A \\
dacriocistografia foi importante para diagnosticar o local da obstrução, o \\
grau de dilatação das vias lacrimais, assim como as alterações de estruturas \\
vizinhas. Estas informações sem dúvida são úteis para o planejamento \\
cirúrgico e como indício prognóstico. \\
\hline
\end{tabular}

Descritores: Obstrução dos ductos lacrimais/radiografia; Técnicas de diagnóstico oftalmológico; Estudos retrospectivos; Adulto
Trabalho realizado na Faculdade de Medicina de Botucatu da Universidade Estadual Paulista - UNESP.

${ }^{1}$ Livre-docente do Departamento de Oftalmologia, Otorrinolaringologia e Cirurgia de Cabeça e Pescoço da Faculdade de Medicina de Botucatu da Universidade Estadual Paulista - UNESP.

${ }^{2}$ Residente de Oftalmologia da Faculdade de Medicina de Botucatu da Universidade Estadual Paulista - UNESP.

${ }^{3}$ Titular do Departamento de Bioestatística da Faculdade de Medicina de Botucatu da Universidade Estadual Paulista - UNESP.

${ }^{4}$ Graduanda(o) da Faculdade de Medicina de Botucatu da Universidade Estadual Paulista - UNESP.

${ }_{5}^{5}$ Médico Radiologista.

Endereço para correspondência: Silvana A. Schellini Distrito Rubião Júnior, SN - Botucatu (SP)

CEP 18610-000

E-mail: sartioli@fmb.unesp.br

Recebido para publicação em 22.01.2004

Versão revisada recebida em 03.06.2004

Aprovação em 23.08.2004

Nota Editorial: Pela análise deste trabalho e por sua anuência na divulgação desta nota, agradecemos às Dras. Suzana Matayoshi e Simone V. Faber Bison.

\section{INTRODUÇÃO}

A semiologia da via lacrimal excretora envolve dados anamnésticos, avaliação funcional e anatômica. A avaliação funcional pode ser feita usando testes com corantes, cintilografia e ressonância magnética. Já, a avaliação anatômica, é realizada pela dacriocistografia (DCG), tomografia computadorizada ou ressonância magnética ${ }^{(1)}$.

A DCG pode determinar com precisão a localização do bloqueio ou estenose da via lacrimal excretora ${ }^{(2)}$. Este é, portanto, um exame muito útil para a avaliação anatômica do sistema, quando se cogita em terapêutica cirúrgica.

O exame dacriocistográfico foi introduzido por Ewing, em 1909; Von Szilly, em 1920, descreveu as vias lacrimais radiograficamente e, em 1964, Campbell melhorou a técnica por magnificação geométrica da imagem ${ }^{(3)}$. Modificações foram descritas, como a de Galloway, em 1984, que introduziu a técnica de subtração digital ${ }^{(4)}$, método especialmente útil quando a DCG deixa dúvida diagnóstica ${ }^{(5)}$.

Basicamente o exame dacriocistográfico representa a imaginologia da via lacrimal por injeção de contraste no interior da mesma, sendo possível empregar contraste hidrossolúvel ou lipossolúvel. O lipossolúvel é de eliminação mais lenta, já que não se mistura à lágrima; porém, delimita muito melhor o contorno das estruturas, sendo melhor para avaliação das vias lacrimais quando se suspeita de tumores, traumatismos e fístulas, pois a imagem obtida é mais precisa ${ }^{(6)}$. 
No Serviço onde foi feito o presente estudo, a DCG é feita de rotina na semiologia das vias lacrimais quando existe suspeita de obstrução da via lacrimal baixa, sendo executada pelo oftalmologista ou tendo a participação dele na sala de exames e o objetivo deste é relatar os achados dacriocistográficos obtidos.

\section{MÉTODOS}

Foi realizado estudo retrospectivo, avaliando-se as DCGs de 100 indivíduos adultos, nos quais se suspeitou de obstrução nasolacrimal (ONL), atendidos na Faculdade de Medicina de Botucatu - UNESP.

Os pacientes foram avaliados quanto a parâmetros clínicos: sexo, faixa etária, queixa e duração do lacrimejamento, história de dacriocistite aguda prévia, antecedentes de trauma da face ou cirurgia nasal e o resultado obtido no exame dacriocistográfico.

Os parâmetros radiológicos avaliados foram: local da obstrução, grau de dilatação do saco lacrimal e sinais indiretos de alterações nasais. $\mathrm{O}$ grau de dilatação do saco lacrimal foi classificado segundo observações pessoais em: grau 0, quando o saco é inexistente ou atrófico; grau 1, quando o saco lacrimal tem as dimensões semelhantes ao saco lacrimal normal; grau 2, quando pequena dilatação está presente; grau 3 quando a dilatação é média e grau 4, quando o saco lacrimal encontra-se bastante dilatado.

O exame foi realizado durante a avaliação semiológica da via lacrimal, para confirmação diagnóstica da ONL.

\section{Descrição do exame}

Dilatação do ponto lacrimal superior ou inferior utilizando dilatador de ponto lacrimal, seguida da cateterização do ducto lacrimal com agulha butterfly $\mathrm{n}-25$, com bisel cortado, acoplada a seringa descartável de $3 \mathrm{ml}$. Introdução 2 a $3 \mathrm{ml}$ do contraste oleoso (Lipiodol ${ }^{\circledR}$-Guerbet), com paciente sentado, posição de mento-naso-placa, sendo obtida uma radiografia logo após a injeção do contraste em ambas as vias lacrimais. A análise radiológica foi feita sempre pelo mesmo examinador.

Os dados clínicos e dacriocistográficos obtidos foram submetidos à análise estatística descritiva (distribuição da freqüência de ocorrência) e teste do qui-quadrado para uma $\operatorname{amostra}^{(7)}$.

\section{RESULTADOS}

Os pacientes, $74,49 \%$ do sexo feminino e $25,51 \%$ do sexo masculino $\left(X^{2}=23,51-p<0,005\right)$, encontravam-se distribuídos principalmente nas faixas etárias de 51 a 60 anos $(22,45 \%)$ e maiores de 60 anos $(45,92 \%)\left(X^{2}=71,96-p<0,005\right)$.

A queixa de epífora estava ausente em $3,0 \%$. Em 32,0\% a queixa estava presente há cerca de 1 ano; em $37,0 \%$ entre 1 a 5 anos e em 28,0\%, há mais de 5 anos $\left(X^{2}=27,63-p<0,0001\right)$ (Tabela 1).

A dacriocistite aguda prévia havia ocorrido em 4 pacientes $(4,08 \%)\left(X^{2}=76,94-p<0,0001\right)$.

Apresentavam antecedentes de trauma de face $3(3,06 \%)$ pacientes $\left(X^{2}=240,69-\mathrm{p}<0,0001\right)$, e $6(6,1 \%)\left(X^{2}=75,47\right.$ $\mathrm{p}<0,005)$, haviam sido submetidos à cirurgia nasal previamente.

A avaliação do exame dacriocistográfico de 19 pacientes teve diagnóstico inconclusivo por se ter qualidade técnica insatisfatória.

A avaliação das vias lacrimais revelou sinais de obstrução alta em 9,04\%, obstrução baixa em $38,42 \%$, sendo que em $34,46 \%$ o local da obstrução era o seio de Arlt e em 3,96\%, o ducto nasolacrimal. Cerca de $33,33 \%$ dos exames evidenciaram vias lacrimais pérveas sem dilatação e $19,21 \%$, vias pérveas e dilatadas $\left(X^{2}=67,72\right.$ - $\left.p<0,001\right)$ (Gráfico 1).

A classificação do grau de dilatação dos sacos lacrimais foi realizada em 75 do total de exames avaliados. Cerca de $4,00 \%$ tiveram o saco lacrimal grau $0,21,33 \%$ grau $1,38,67 \%$ grau $2,34,67 \%$ grau 3 e $1,33 \%$ grau $4\left(X^{2}=43,87-p<0,0001\right)$ (Gráfico 2).

Cerca de $33,67 \%$ dos pacientes apresentavam desvio de septo $\left(X^{2}=10,45-p<0,005\right), 67,35 \%$ hipertrofia de cornetos $\left(\mathrm{X}^{2}=11,80-\mathrm{p}<0,005\right), 4,08 \%$ sinais radiológicos de sinusopatia $\left(X^{2}=82,65-p<0,005\right)$ e em 3,06\% evidenciaram-se alterações ósseas (Gráfico 3).

\begin{tabular}{|c|c|c|}
\hline 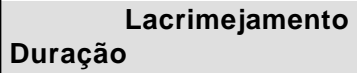 & Pacientes & Freqüência (\%) \\
\hline$<1$ ano & 32 & 32,00 \\
\hline 1 a 5 anos & 37 & 37,00 \\
\hline$>5$ anos & 28 & 28,00 \\
\hline Ausente & 3 & 3,00 \\
\hline$x^{2}=27,63(p<0,0001)$ & & \\
\hline
\end{tabular}

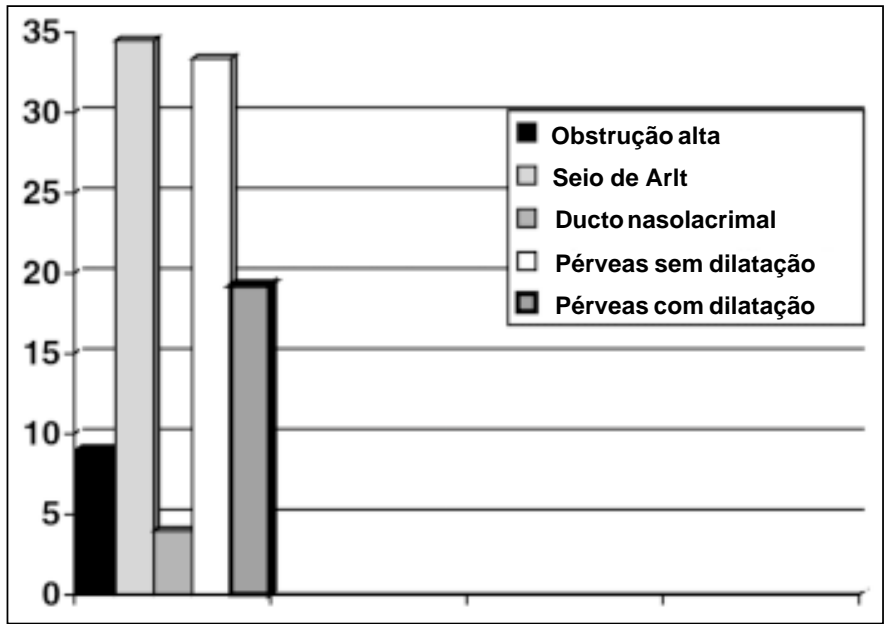

Gráfico 1 - Distribuição dos resultados das dacriocistografias realizadas 


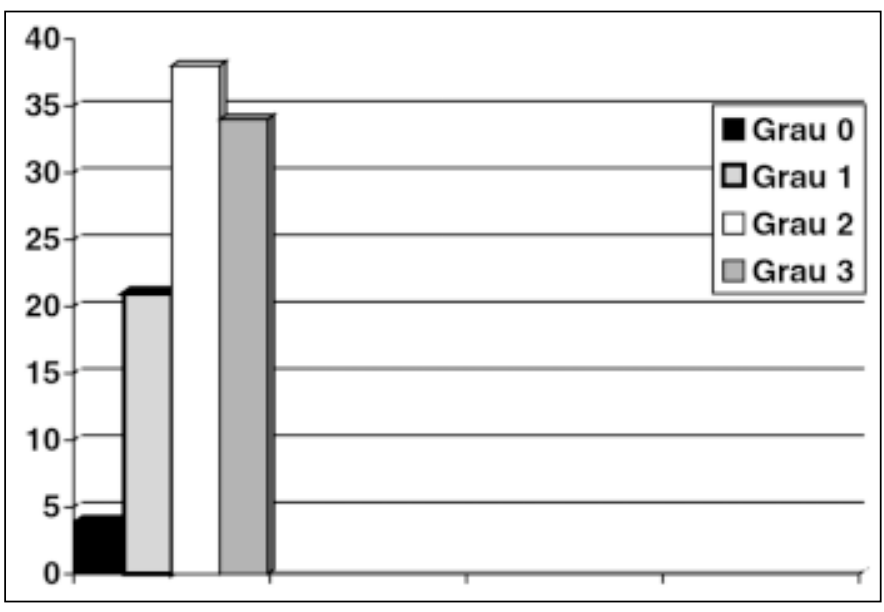

Gráfico 2 - Distribuição dos sacos lacrimais segundo o grau de dilatação

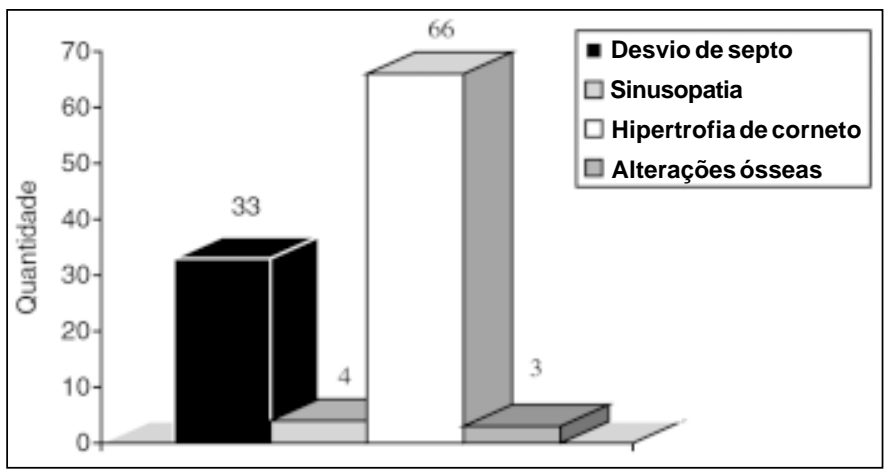

Gráfico 3 - Distribuição de alterações nasais encontradas nos pacientes submetidos à dacriocistografia

\section{DISCUSSÃO}

A maioria dos indivíduos $(74,49 \%)$ sob suspeita de ONL era do sexo feminino. A dacrioestenose é mais freqüente no sexo feminino, constatação já feita por outros autores que citaram pertencerem ao sexo feminino 62 a $85 \%$ dos pacientes $\operatorname{afetados}^{(6,8)}$. Uma das hipóteses para a maior ocorrência de obstrução em mulheres seria devido ao fato de que as mulheres possuem menor diâmetro da porção intra-óssea do canal nasolacrimal ${ }^{(9)}$.

A maior parte dos pacientes encontrava-se com idade superior a 60 anos, embora seja conhecida a tendência da obstrução afetar principalmente mulheres na terceira década de $\operatorname{vida}^{(5)}$.

Quanto à duração da epífora, foi semelhante o número de pacientes com queixa há menos de 1 ano, de 1 a 5 anos e há mais de 5 anos, em ambos os olhos. Muitos pacientes não conseguiram precisar a época do aparecimento da epífora, provavelmente por se tratar de quadros crônicos e de instalação não abrupta. Procurou-se avaliar se a duração da epífora influenciaria o grau de dilatação do saco lacrimal, o que não se confirmou.

A maioria dos pacientes não apresentava história prévia de dacriocistite aguda e poucos apresentaram trauma da face ou cirurgia nasal previamente.

Quanto aos resultados dacriocistográficos, é conduta do serviço ter apenas uma imagem após a injeção do contraste. Com isso, o paciente é exposto à quantidade menor de radiação, além do que, observações pessoais permitiram saber que o contraste oleoso impregna as paredes da via lacrimal, permanecendo na via excretora, mesmo que normal, por tempo que pode ser superior a 15 ou 30 minutos que são os tempos preconizados para a chapa de retardo.

No presente estudo, a obstrução baixa, localizada o mais das vezes no seio de Arlt, ou vias pérveas e sem dilatação, foram os achados mais comuns. A obstrução alta, além de infreqüente, não requer avaliação por DCG, uma vez que a cateterização dos canalículos já confirma o diagnóstico.

As vias pérveas e dilatadas aqui encontradas são prova de que a estase pode ocorrer em condições em que não existe bloqueio anatômico, tais como flacidez palpebral, ectrópio, paralisia facial, lacrimejamento reflexo (olho seco, alergias). Mesmo em crianças, a presença de vias pérveas nos exames dacriocistográficos é achado importante, definindo a não existência anatômica de obstrução das vias lacrimais ou de pseudoobstrução ${ }^{(10-11)}$.

As obstruções da via lacrimal na infância ocorrem, em geral, na válvula de Hasner ${ }^{(10)}$. Segundo os resultados obtidos no presente estudo, nos adultos, a grande maioria das estenoses está localizada na região do seio de Arlt, local situado imediatamente abaixo do saco lacrimal, na região de transição entre o saco e o ducto nasolacrimal.

Nos indivíduos em que o saco lacrimal foi classificado, observou-se que a maioria apresentava saco lacrimal grau 2 ou 3 . O grau de dilatação do saco lacrimal influencia na indicação terapêutica, uma vez que nos portadores de sacos lacrimais atróficos e pequenos (graus 0 e 1), a dacriocistorrinostomia deve ser feita usando entubação das vias lacrimais, já que não há como se construir uma "ponte" entre o saco lacrimal e a mucosa nasal. Quando existe grande dilatação do saco (grau 4), há necessidade de se ressecar mais mucosa do saco lacrimal com o intuito de não se deixar após o procedimento uma mucocele que continuará represando lágrima e propiciando a instalação do processo de inflamação crônica da via lacrimal excretora. Também para a realização da dacriocistorrinostomia pela via endoscópica, o saco lacrimal não deve ser atrófico, pela dificuldade de se localizar a estrutura. Portanto, a DCG fornece importante informação previamente ao ato cirúrgico, permitindo que se tenha à mão materiais que serão indispensáveis para o resultado cirúrgico ou que se possa melhor optar por procedimentos cirúrgicos mais adequados.

A maioria dos pacientes não apresentou desvio de septo, nem sinusopatia ou alteração óssea. Mas, em grande parte, observou-se hipertrofia de corneto, condição que pode levar a dificuldade para a progressão da lágrima, estase, dilatação e infecção das vias lacrimais excretoras ${ }^{(11)}$. Estas alterações nasais que podem ser evidenciadas com a DCG, podem também indicar que existe necessidade de avaliação mais pormenoriza- 
da da cavidade nasal e que poderão existir dificuldades técnicas durante a execução da dacriocistorrinostomia.

Além da DCG, pode-se usar na propedêutica outras variantes deste exame, como a macrodacriocistografia e a DCG com subtração digital $^{(12)}$, capazes de trazer ainda melhor acurácia à imagem radiológica, facilitando a visibilização de alterações da via lacrimal excretora.

Concluindo, a DCG permite diagnosticar o local da obstrução, o grau de dilatação das vias lacrimais, assim como as alterações de estruturas vizinhas, sendo parte importante da semiologia das vias lacrimais excretoras, possibilitando uma programação cirúrgica adequada e previsão do prognóstico pós-operatório.

\section{AGRADECIMENTO}

Os autores agradecem ao pós-graduando Carlos Roberto Pereira Padovani pela tabulação dos dados para análise estatística e ao Dr. Fernando Gonçalves pelo auxílio na confecção dos gráficos deste estudo.

\section{ABSTRACT}

Purpose: To evaluate dacrycystographic examination in patients with suspicion of lacrimal apparatus obstruction. Methods: A retrospective study was performed to evaluate dacryocystographies of 100 adults suspected of nasolacrimal duct obstruction at the "Faculdade de Medicina de Botucatu - UNESP". The data were submitted to descriptive statistical analysis and chi-square test. Results: The patients were mainly female, over 60 years old, presenting a low obstruction located at Arlt's sinus, with a grade 2 or 3 lacrimal sac. Lacrimal pathways with normal tear drainage, with or without dilatation and nasal alterations such as concha hypertrophy were also observed. Conclusion: Dacryocystographic evaluation was important to show the level of obstruction, the presence of dilatation of the lacrimal sac, as well as alterations in nearby structure. This information might influence the adopted surgical technique and the final treatment results.

Keywords: Lacrimal duct obstruction/radiography; Diagnostic techniques, ophthalmological; Retrospective studies; Adult

\section{REFERÊNCIAS}

1. Costa MN, Schellini SA, Moura EM. Conjuntivodacriocistorrinostomia. In: Soares EJC, Moura EM, Gonçalves JOR. Cirurgia plástica ocular. São Paulo: Rocca; 1997. v8. p.263-70.

2. Lloyd GA, Welham RA. Substraction macrodacryocystography. Br J Radiol. 1974;47(559):379-82.

3. Campbell W. The radiology of the lacrimal system. Br J Radiol. 1964;37(1):1-26.

4. Galloway JE, Karvic TA, Raflo GT. Digital substraction macrodacryocystography. A new method of lacrimal system imaging. Ophthalmology. 1984;91 (8):956-62.

5. Sanmartin ZJ. Dacriocistografia com subtração digital (DGGSD). Arq Bras Oftalmol. 1998;61(2):224-8.

6. Weil B. Vias lacrimais. An Oftalmol. 1988;8(1):26-32.

7. Norman GR, Streiner DL. Biostatistics: the bare essentials. St Louis: Mosby; 1994. p. 260

8. Castrén JA, Korhonen M. Significance of dacryocystography in lacrimal drainage system affections. Acta Ophthalmol. 1963;41:188-92.

9. Janssen AG, Mansour K, Bos JJ, Castelijns JA. Diameter of the bony lacrimal canal: normal values and values related to nasolacrimal duct obstruction: assessment with CT. AJNR. Am J Neuroradiol. 2001;22(5):845-50.

10. Soares EJC, França VP. Importância da dacriocistografia no tratamento da obstrução lacrimal do recém-nascido. Arq Bras Oftalmol. 1987;50(6):225-36.

11. Schellini SA, Silva MRBM, Schellini RC. Pseudo-obstrução nasolacrimal na infância. Arq Bras Oftalmol. 1994;57(5):348-51.

12. Montanara A, Mannino G, Contestabile MT. Macrodacryocystography and echography in diagnosis of disorders of the lacrimal pathways. Surv Ophthalmol. $1983 ; 28(1): 33-41$.

\title{
XXX Simpósio da Associação Paranaense de Oftalmologia
}

\section{3 a 25 de Junho de 2005 \\ Bourbon Curitiba Hotel - Curitiba - PR Tema Oficial: Qualidade de Visão}

\author{
INFORMAÇÕ ES: Creative Solution \\ Tel: (11) 5575-0254 - Fax (11) 5539-2186 \\ E-mail: congressoapo@ terra.com.br
}

